\title{
"LE DOMAINE DE LA TERRE», UN CONJUNTO DE REALIZACIONES-PILOTO A BASE DE TIERRA, EN L'ISLE D'ABEAU (FRANCIA)
}

("LE DOMAINE DE LA TERRE», an ensemble of pilot constructions made of earth in L'Isle D'Abeau [France])

Traducción, adaptación y material gráfico J. Salas y R. Moreno.

\section{RESUMEN}

Se presentan en el trabajo de forma sistematizada algunas de las realizaciones de viviendas experimentales a base de tierra (bloques, tapial, bloques compactados, tierra-paja...) realizadas en las cercanias de Lyon (Francia) y que constituyen el conjunto "Le Domaine de la Terre". Se trata de una realización a base de once proyectos distintos en los que han intervenido un buen número de instituciones, equipos de proyecto $y$ profesionales franceses. Globalmente constituyen una excelente exposición a escala natural de las posibilidades de las construcciones de tierra, aunque los resultados son muy diversos -formal y conceptualmente- entre los distintos proyectos.

\section{SUMMARY}

In this work, some of the experimental housing works (blocks, formwork, mud and straw...) built on the outskirts of Lyon (France) are presented in a systematic way. The ensemble is called "Le Domaine de la Terre", and eleven different projects are concerned, where a number of institutions and teams of professionals have worked. In its whole it is an excelent exposition at natural scale of the possibilities of mud construction, even though the results are of a great diversity -formal and conceptually- between those diverse projects.

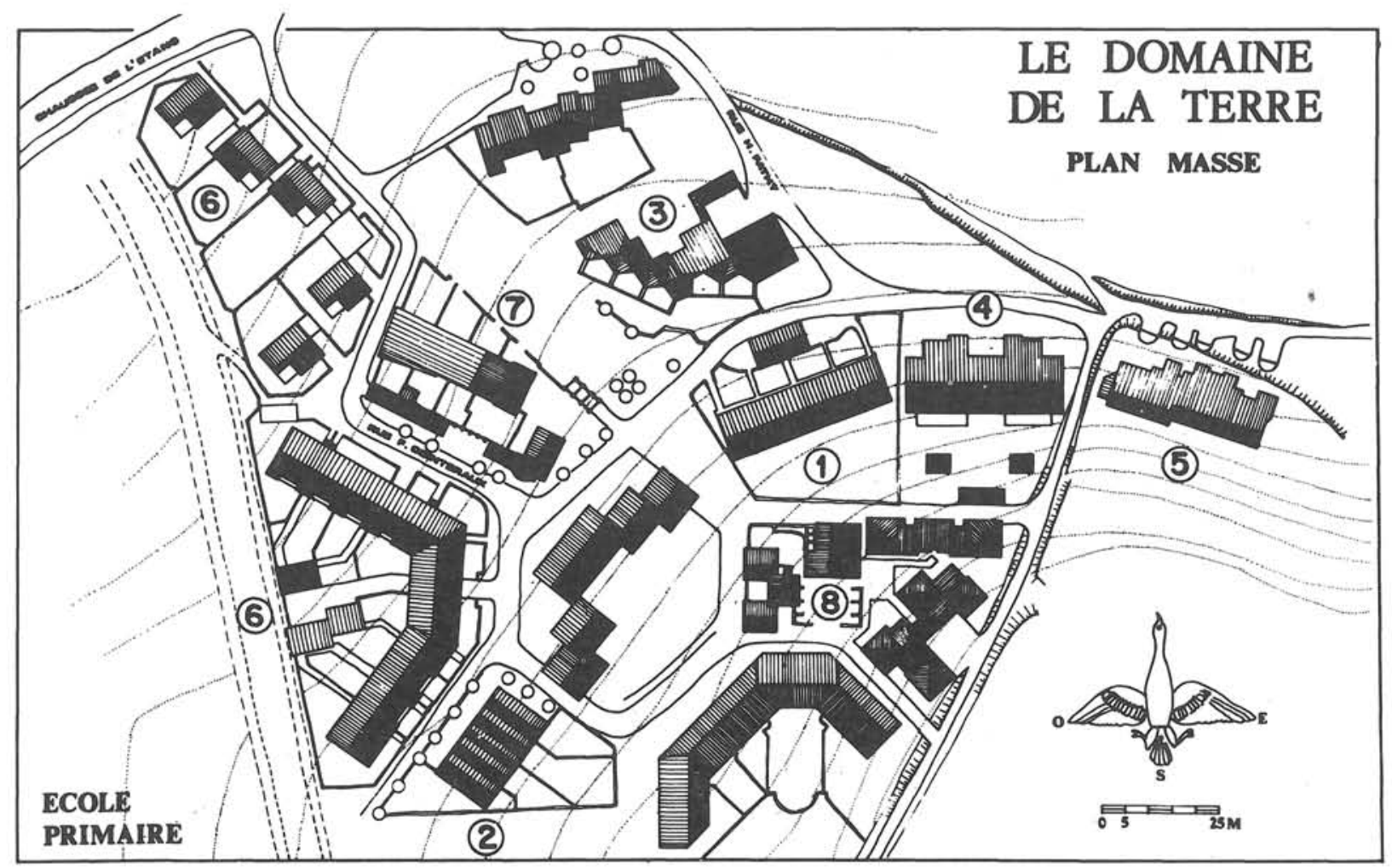




\section{INTRODUCCION}

Los textos que siguen, son traducción parcial del trabajo que, con el titulo "Le Domaine de la Terre", publicó la revista francesa "H» en su n. 0111 , correspondiente a octubre de 1985. Los alzados que se reproducen, corresponden igualmente a la citada publicación. La selección de proyectos, traducción de los textos y el material fotográfico que sigue, es la modesta aportación de los presentadores de esta realización, con la intención de difundir entre los lectores de INFORMES, este conjunto de proyectos ejecutados a base de tierra en algunas de sus múltiples modalidades tecnológicas.

El conjunto de realizaciones que presenta este trabajo, es el resultado de un largo y colectivo esfuerzo en el que han participado, de una u otra manera un buen número de instituciones y profesionales franceses. A la construcción de "Le Domaine de la Terre" han contribuido: el Centro Pompidou, el C.C.I., CRATERRE, el CSTB, la Escuela de Arquitectura de Grenoble, la E.N.T.P.E. de Lyon, el Plan Construction, SOCOTEC..., por sólo mencionar unas cuantas entidades, asi como once equipos de arquitectos, quince empresas constructoras y numerosas empresas de ingenieria e industrias afines.

Al pie de la página anterior se reproduce el plano general de situación de la realización, sobre el que se marca el orden de descripción de los distintos proyectos seleccionados y que siguen en páginas sucesivas.

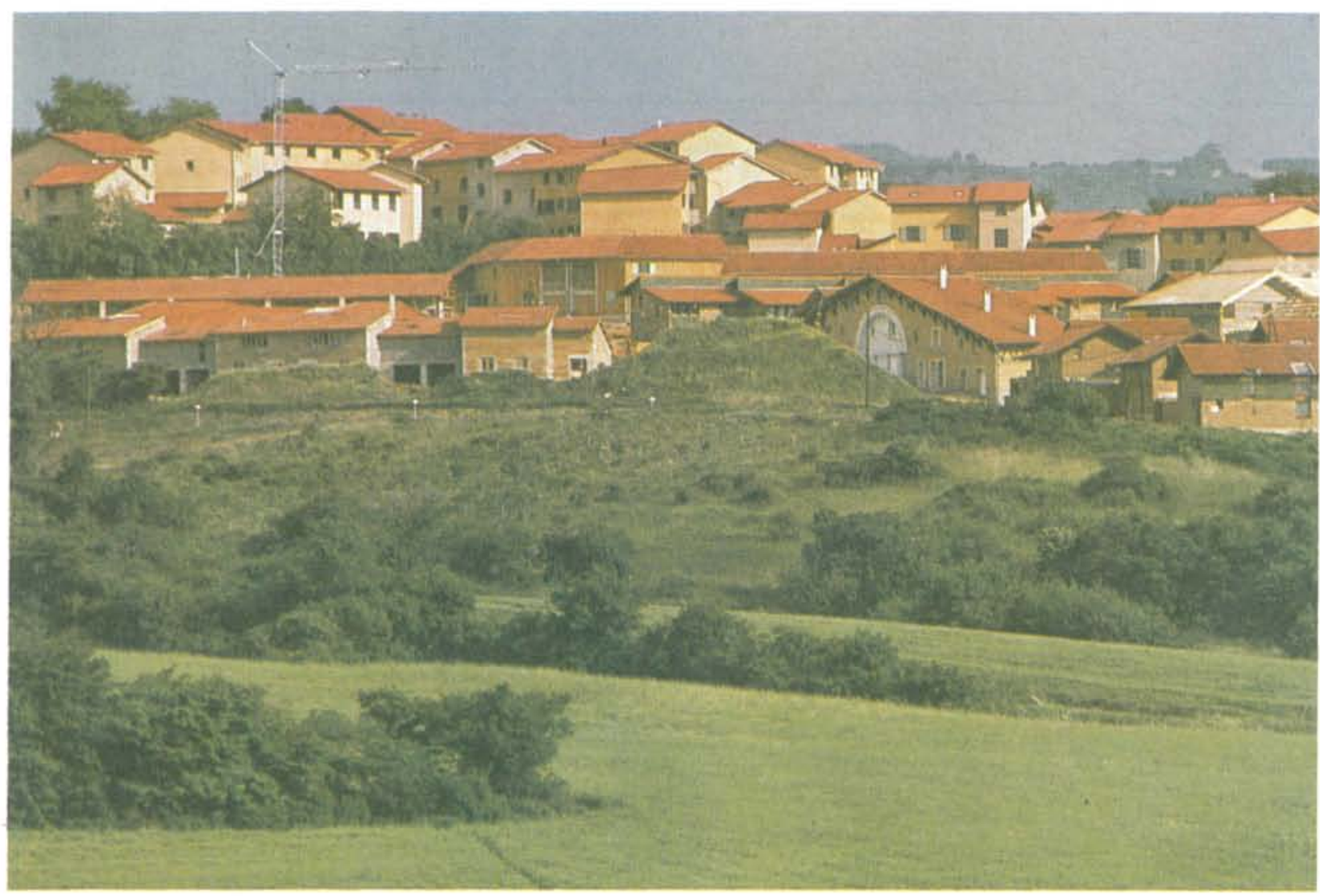


(1)

\section{CUATRO VIVIENDAS EN BANDA DE TAPIAL NO ESTABILIZADO}

Arquitectos: Serge Jaure, François Confine, Jean Pierre Duval

Un proyecto de rasgos simples, que se caracteriza por su apariencia inspirada en algunos hangares agricolas.

La idea básica es construir un "paraguas" (el techo), antes que los muros. Bajo este techo a dos aguas, sostenido por un armazón de madera, las casas adheridas albergan dos niveles de clara tipologia: zona diurna en la planta baja y zona nocturna en la planta superior. Esta producción fue muy apreciada durante la obra, a menudo afectada por las lluvias. Esta ventaja pudo ser a veces un inconveniente, especialmente en lo concerniente al acabado del tapial bajo la techumbre.

La disociación de la función del soporte entre la estructura de madera que sostiene el techo y los muros en tapial que sujetan el forjado, lleva a una doble lectura en la fachada: una estructura de connotaciones algo industriales, se integra en una construcción tradicional.

Este edificio, que ha sido frecuentemente considerado como uno de los más espectaculares de la obra, denota una sobria elegancia.

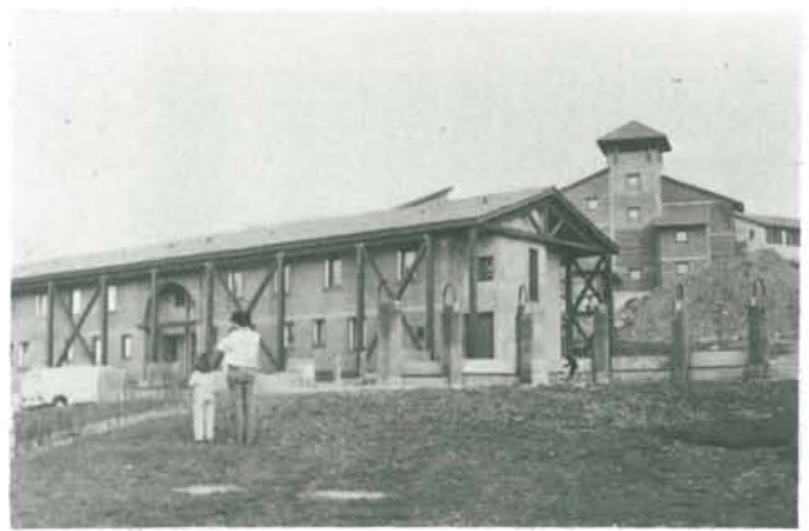

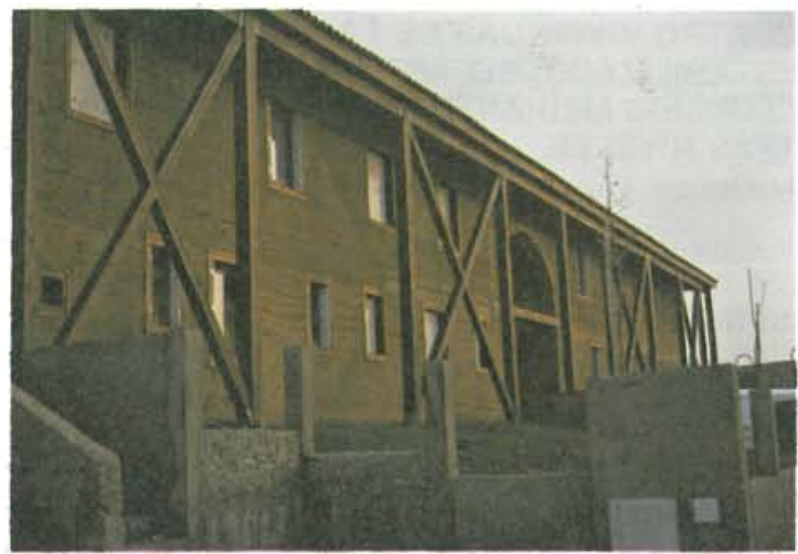

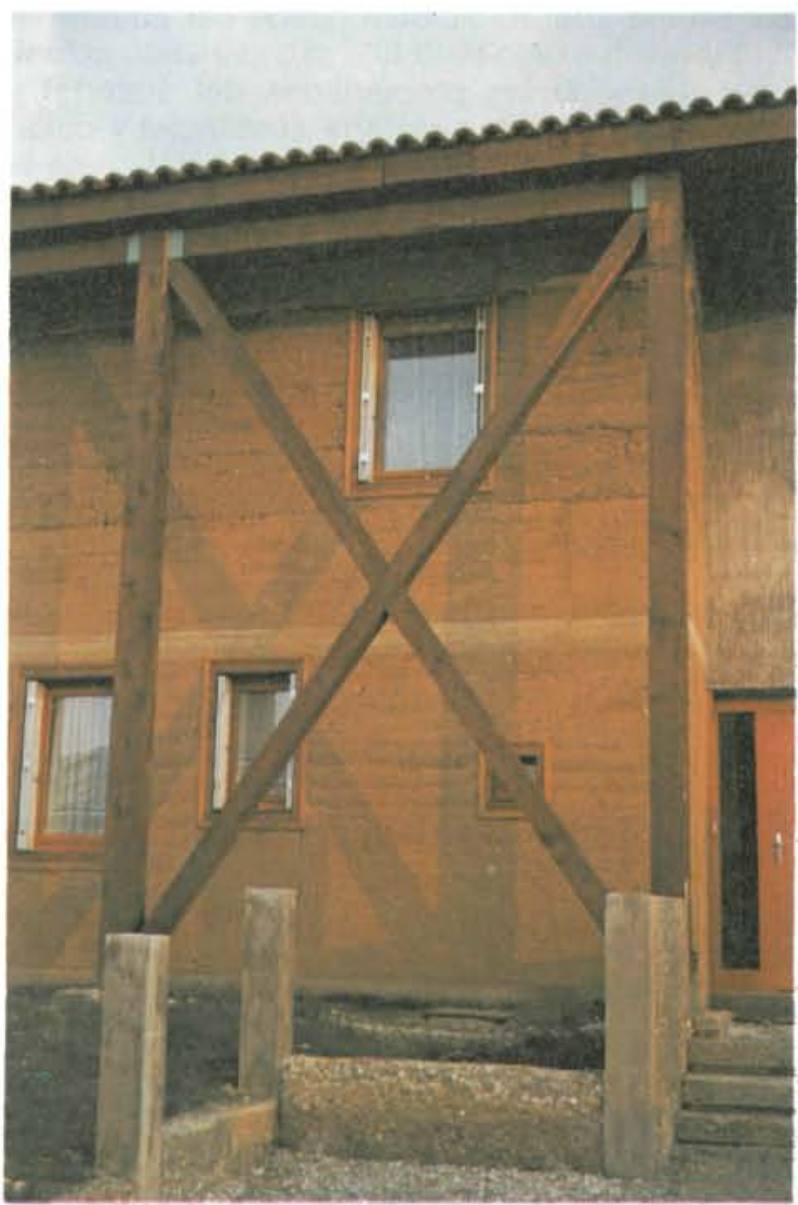

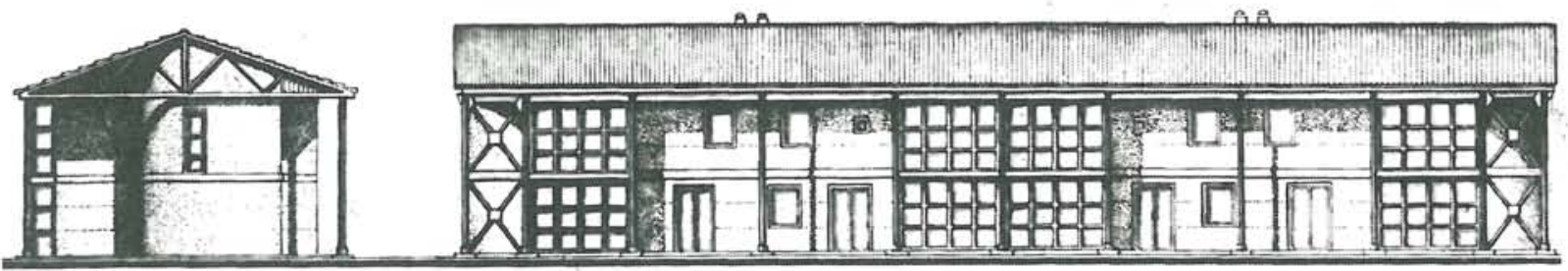




\section{CUATRO VIVIENDAS DE TAPIAL NO ESTABILIZADO, REPARTIDAS EN DOS EDIFICIOS MEDIANEROS DE TRES NIVELES}

Arquitectos: Françoise Jourda, Gilles Perraudin

Los arquitectos lyoneses exponen claramente la parte arquitectónica: "Este proyecto rechaza la disyuntiva modernista-internacional v/s historicista-cultural. Pretende mostrar que la única via para un anclaje cultural efectivo, pasa por un modo de pensar tradicional, es decir, racionalista. Es asi como la rigurosidad de trabajo y expresión dan un resultado que asemeja tanto al megarón miceniano como a los hangares agricolas, ambos arquetipos de origen. Se trata, pues, de una arquitectura vernácula - internacional».

El otro deseo de los arquitectos fue demostrar que un material tradicional puede ser puesto en obra y revalorizado para una problemática actual. Esto utilizando las propiedades del material y hacièndolas resaltar: materia sustancial y color, masa térmica, capacidad de carga estática de la tierra. La concepción de la estructura simple demuestra una perfecta asimilación de los desempeños del material y de la lógica de producción que le es propia.

Este rigor ha permitido una optimizacion, tanto económica, como técnica expuesta por el proyecto y el gusto de un trabajo soñado por la empresa. Resultado de un diálogo permanente entre los asociados.

Este proyecto, muy logrado, expresa claramente una nueva modernidad de la tierra y el tapial.

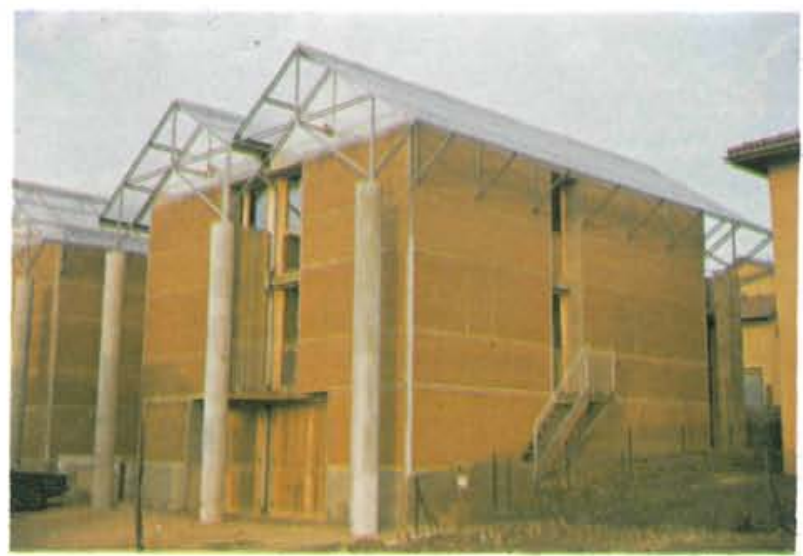

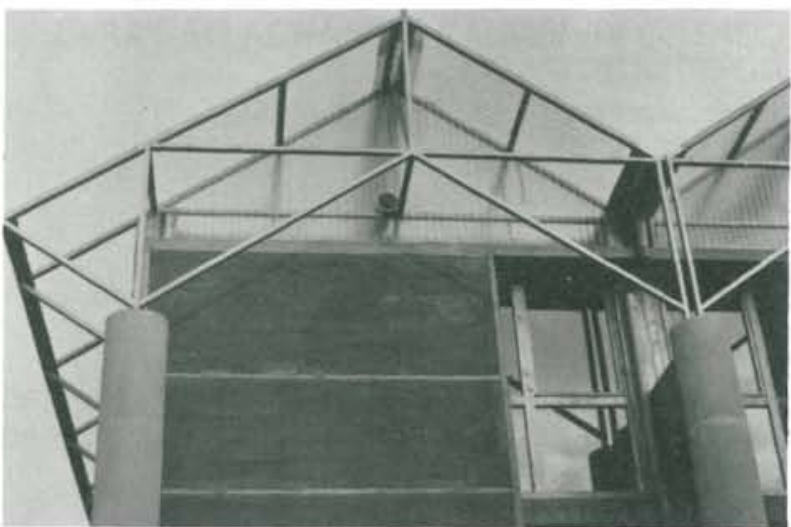
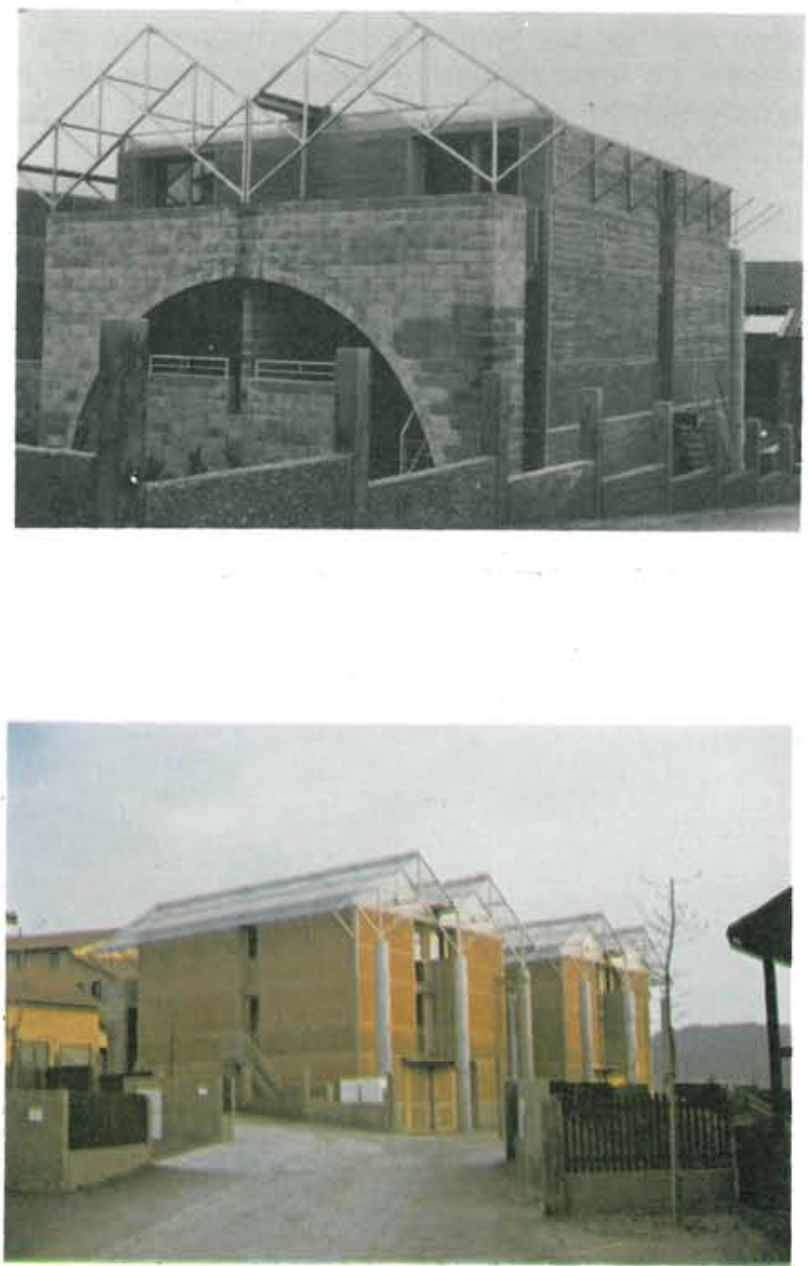

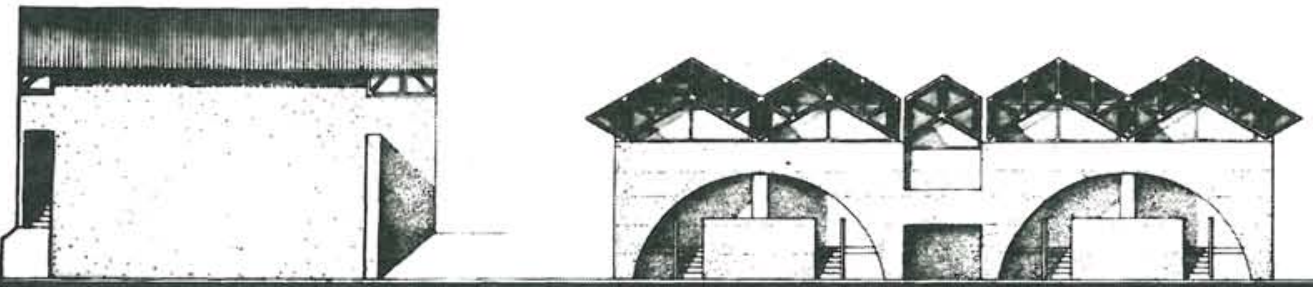




\section{3}

\section{CINCO VIVIENDAS MEDIANERAS CON BLOQUES DE TIERRA VIBRO-COMPACTADA Y ESTABILIZADA}

Arquitectos: Uriel Moch, Georges Chavanne y Patrice Carle

«Nuestra proposición inicial, con respecto a la tierra, era la siguiente: no confundir Francia con los paises en desarrollo, con lógicas economias diferentes. Hacer un experimento basado en la situación actual y utilizar la tierra sólo para rendimientos ventajosos en precio y utilidad, sobre otros materiales... Esto nos lleva a utilizar la tierra como masa térmica, especialmente en los fondos de los invernaderos y en los tabiques".

Nuestro proyecto es más bien un "proyecto térmico" que un "proyecto - tierra». Está basado en la inercia térmica de los edificios y el precalentamiento del aire nuevo por extracción, desde un invernadero y luego inyección en las habitaciones principales.

Habría que hacer un balance del ahorro de energia y también de los problemas de confort en verano, después de la puestas de sol... La generalización de las enseñanzas que se podrian sacar, es muy dificil. Pero no hemos buscado, ni una solución universal, ni una demostración, y es, tal vez, este comienzo de optimización de un caso particular, lo más cercano al comienzo detenido del «hábitat" vernáculo de la tierra.

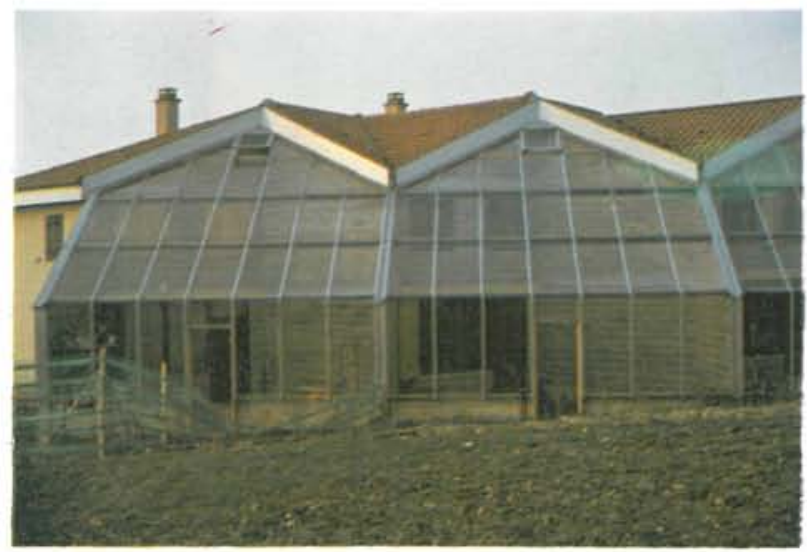

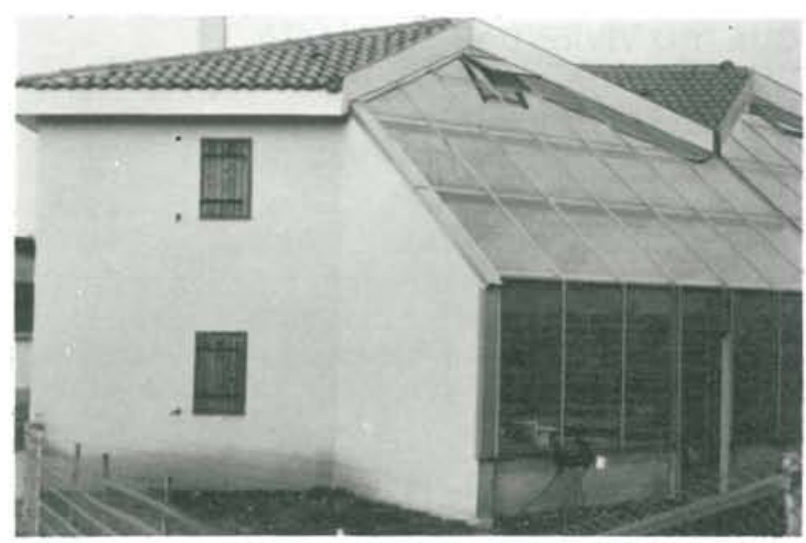

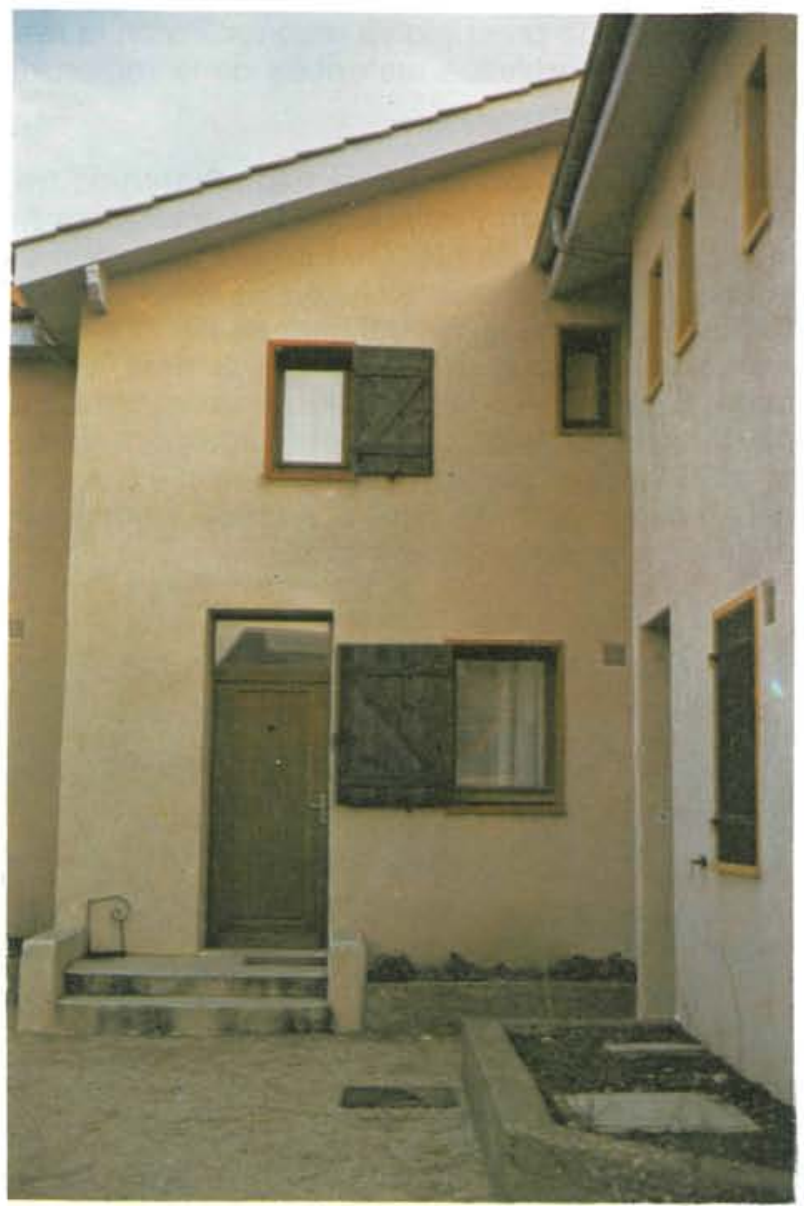

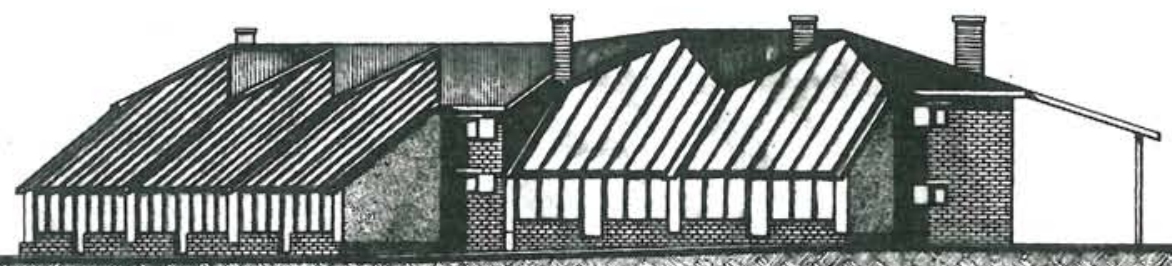

"3." 
(4)

\section{CUATRO VIVIENDAS EN BANDA CONSTRUIDAS CON TAPIAL NO ESTABILIZADO}

Arquitecto: Jean-Vincent Berlottier

La idea es crear un "palacio autárquico" como idea base del proyecto. Aunque dificil de realizar en el plano de las funciones del "hábitat", por razones económicas, esta idea llega a sus mejores resultados en el intento de relacionar las empresas locales con el saber hacer del tapial. La empresa ha realizado un trabajo cuidado que proviene de un prestigio ya reconocido en la restauración de edificios históricos de la región de Brest.

Los encofrados del tapial se han mantenido tradicionales y sólo la compactación neumática ha significado un adelanto. Con una concepción de largos paños de muros, la ejecución fue fácil. Cabe destacar que los volúmenes cilindricos de las cajas de escaleras, quedan a la vista, dando asi a la tierra todo el sentido de la materia y la plasticidad. El edificio está pensado para limitar al máximo el empleo de hormigón, con excepción de las cimentaciones. Tierra, madera y sol cohabitan en una tradición renovada.

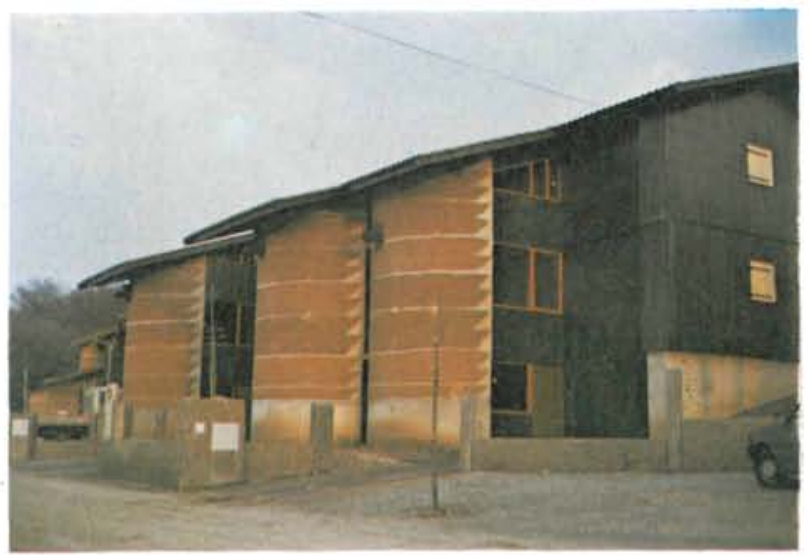

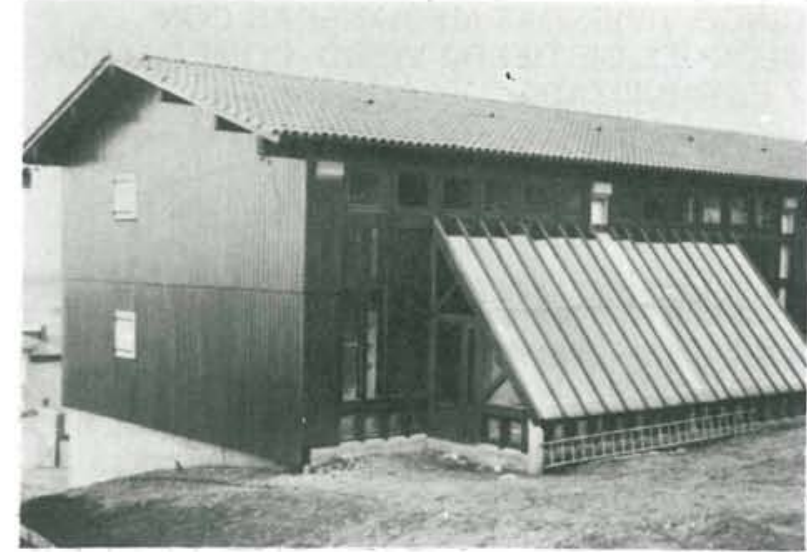
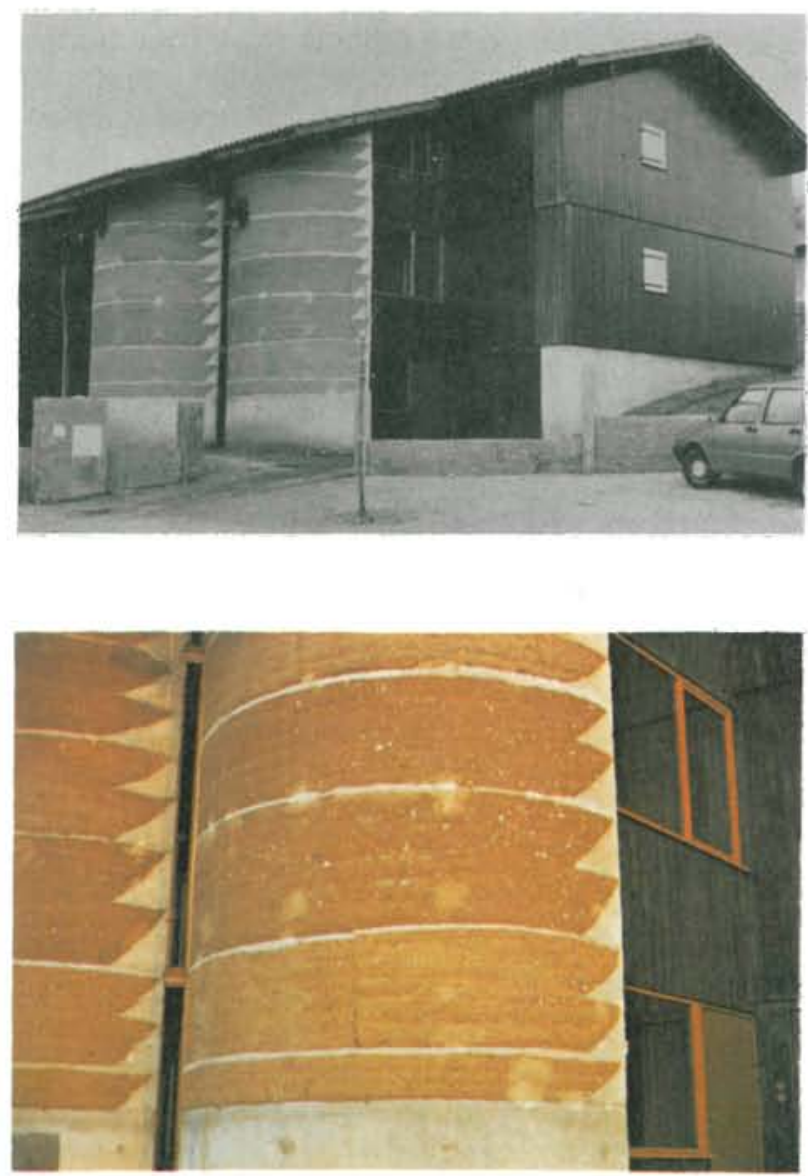

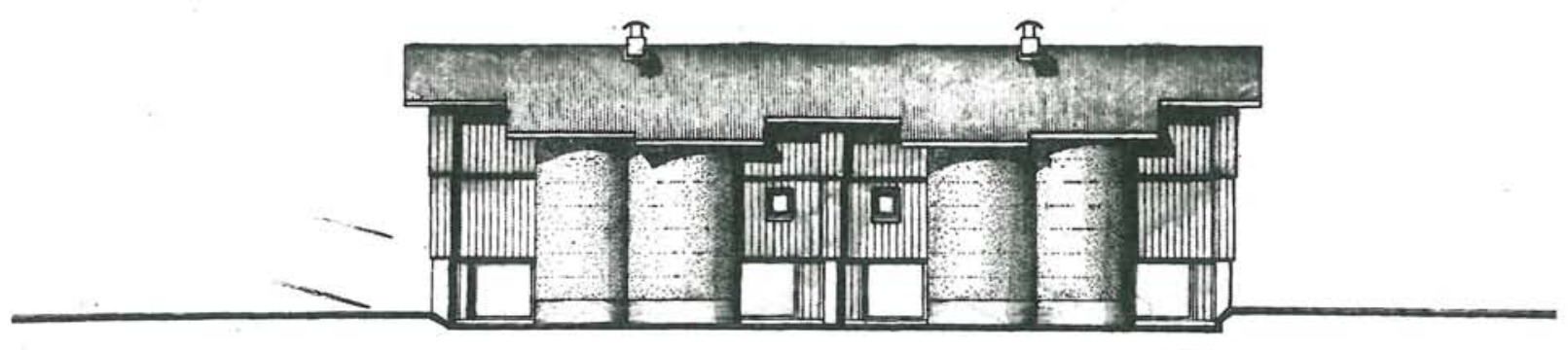

NORD 
5

\section{CUATRO VIVIENDAS EN BANDA, BLOQUES DE TIERRA VIBRO-COMPACTADA Y ESTABILIZADA}

Arquitectos: Dominique Goise (Grupo "Ersol»)

La idea inicial es considerar la tierra en toda la dimensión de trabajo del edificio: relación entre la habitación y los espacios exteriores, expresada por el empleo de técnicas de obras públicas (tierra armada y "conducciones" para la construcción de garajes y la ejecución de cimentaciones), aprovechamiento de los movimientos de tierra de los terraplenes, empleo del adobe en los muros del soporte.

Pero estas ideas iniciales han debido abandonarse. La tierra está finalmente protegida exteriormente por un entablado de madera e interiormente por un enlucido de cal. El proyecto se abre al sur, a pesar de una topografia ingrata y explota la presencia solar con un doble sistema de vidrio y "muros Trombe». El aislamiento exterior optimiza el funcionamiento térmico de los muros de bloques de tierra maciza, que constituyen reservas de calorias.

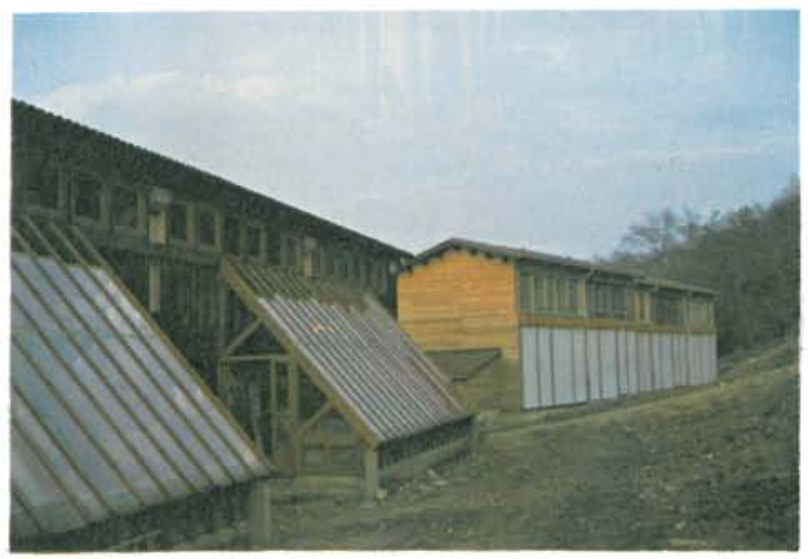

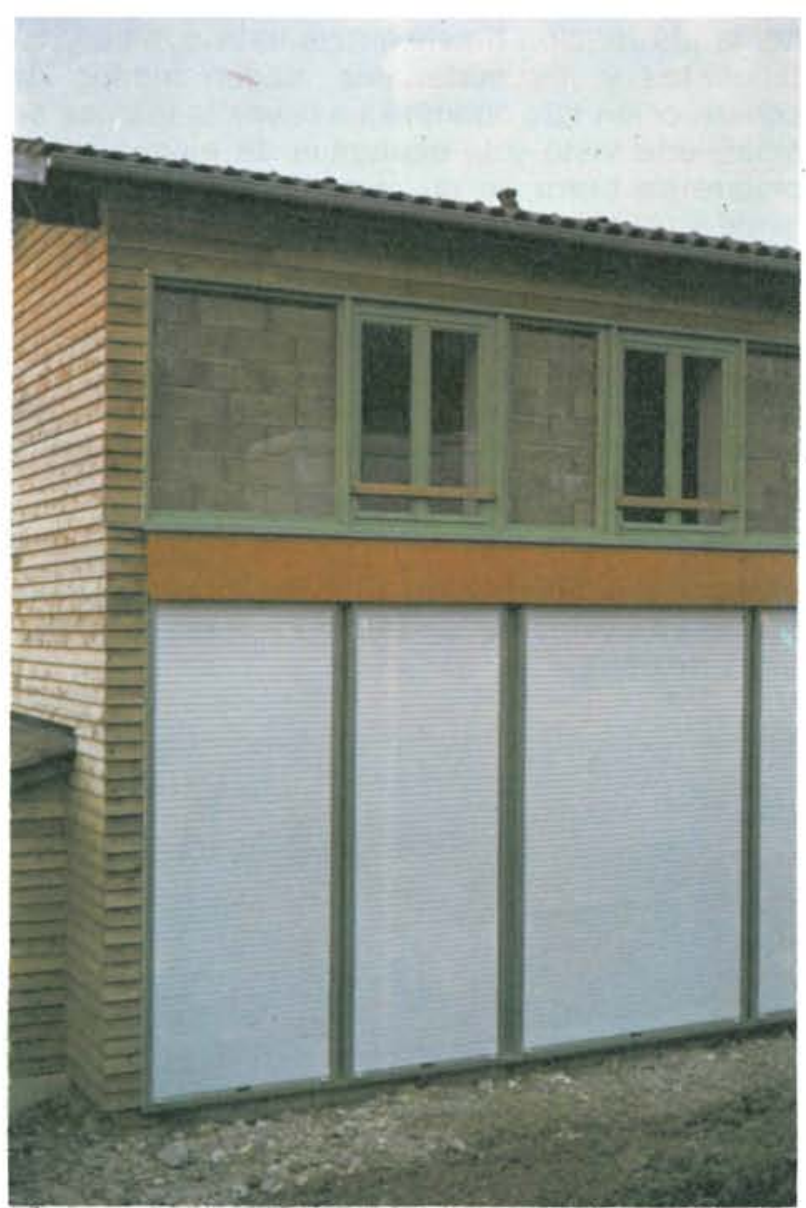

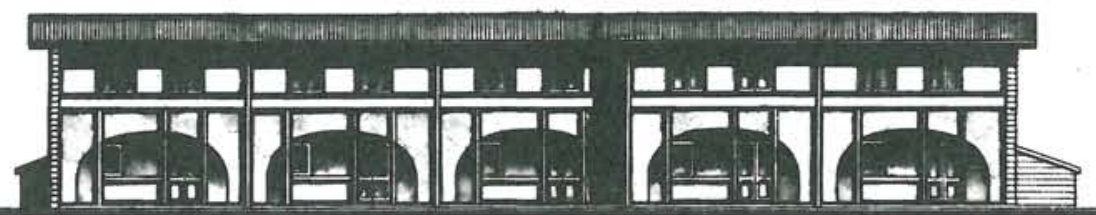




\section{QUINCE VIVIENDAS REPARTIDAS EN CASAS INDIVIDUALES MEDIANERAS EN BLOQUES DE TIERRA VIBRO-COMPACTADA Y EST.ABILIZADA}

Arquitectos: François Galard y Laurence Guibert (Taller «85")

"Cuales quiera que sean las arquitecturas, las formas, las funciones del edificio, si se utiliza la tierra, ésta debe estar a la vista para poder explotar la riqueza de sus tintes, sus diferentes texturas y esa armonia instantánea que se establece gracias a ella, entre el entorno y lo edificado. La tierra es un material de gran riqueza plástica y estética que hace una llamada a las raices".

Los arquitectos integran las diferentes componentes de un proyecto de arquitectura. Toman en cuenta las orientaciones y pendientes del terreno, la agrupación de viviendas individuales yuxtapuestas y los materiales, según modos de construcción que optimizan a la vez la técnica de albañileria vista y la economia de ejecución. El bloque de tierra es un material plenamente vigente en la actualidad y el esquema adoptado controla la reducción de los sobrecostos. El esqueleto de madera está recubierto por una "piel» de tierra, los dos sistemas están separados por una capa de aire que calienta con la técnica de pared transmisora. Un gran número de expresiones arquitectónicas del bloque de tierra, se han manifestado con claridad.

En este proyecto se conjunta, una técnica actualizada y un ejemplo bien acabado, con argumentos de una evidente modernidad de las arquitecturas de tierra.

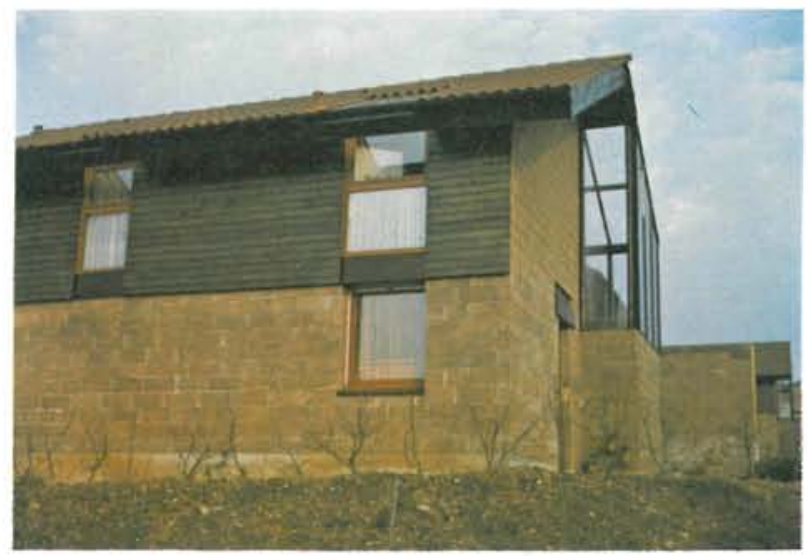

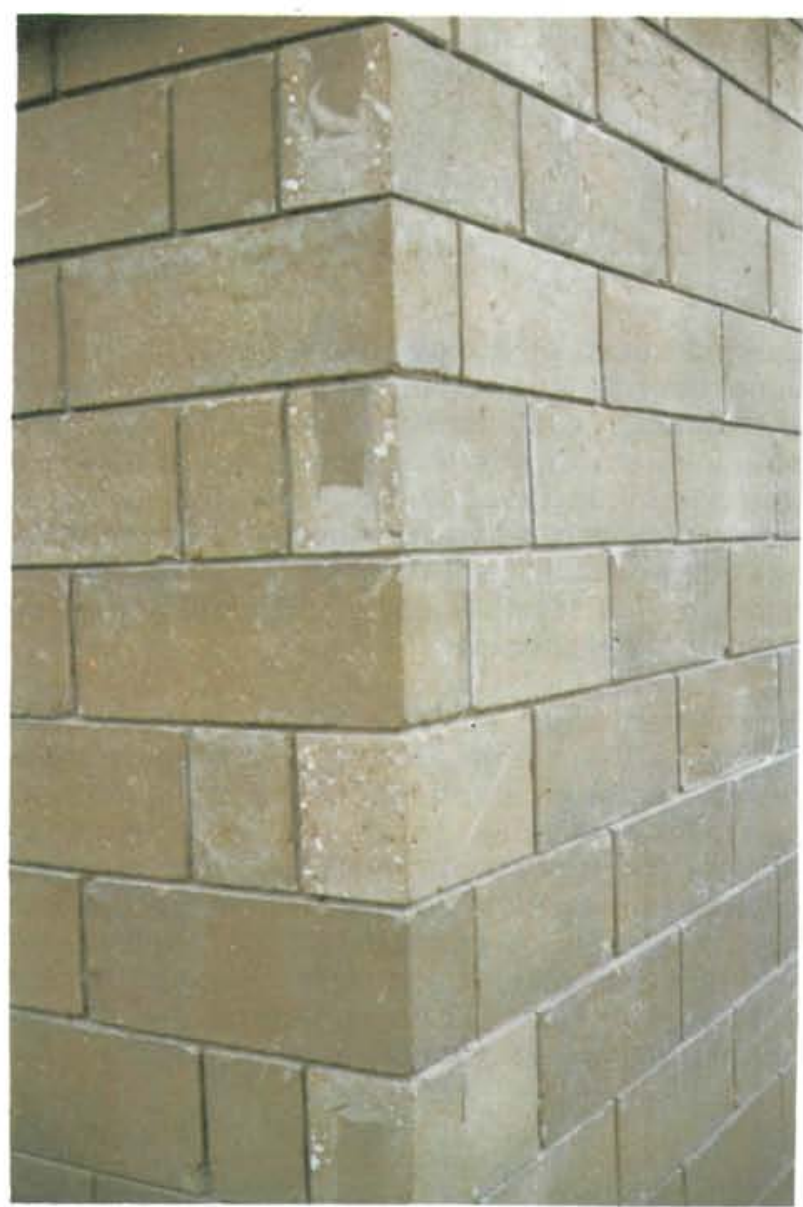

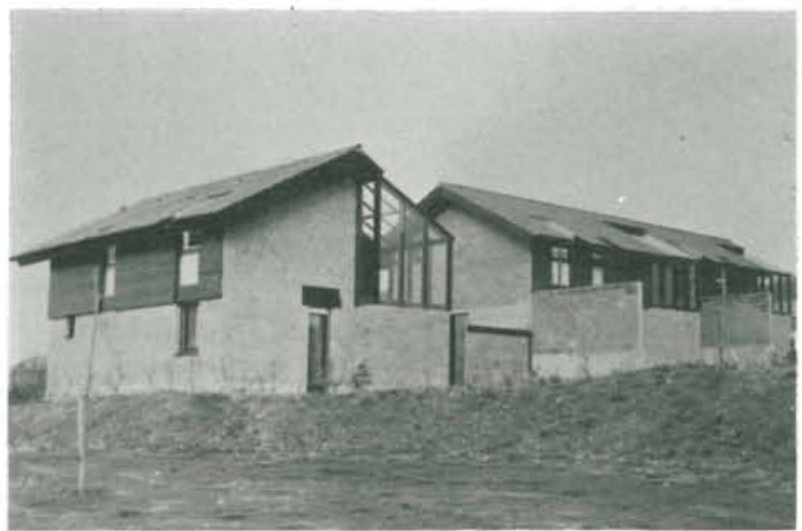

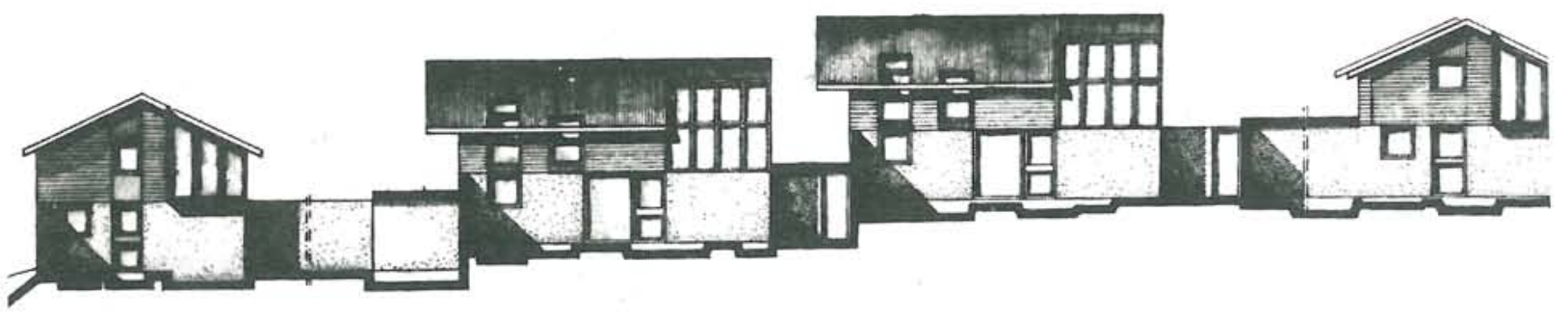




\section{CINCO VIVIENDAS DE TAPIAL TRADICIONAL, REPARTIDAS EN DOS EDIFICIOS, UNO DE ELLOS VIVIENDA INDIVIDUAL}

Arquitectos: Odile Perreau-Hamburger, Jean-Michel Savignat y M. Monteanu

Los arquitectos han propuesto una «reflexión sobre los significados y el vocabulario de una arquitectura de tierra europea y contemporánea". Citando a Odile Perreau-Hamburger: «Existe un placer del material, de su naturaleza pesada, de su grano y color táctil, de su carácter elemental, como el fuego, el agua o el aire. Este placer responde al actual gusto por lo natural (. . .). La economia de su producción y de su costo ecológico, proponen argumentos técnicos precisos para la rehabilitación de la tierra (...). Nos ha chocado la escala de los edificios del Dauphiné. La masa de los muros y los techos anchos y salientes, contrastan con las empalizadas, balaustradas y pértigas (...). Los muros imponentes, las barandas macizas se acoplan a un vocabulario clásico de balcones, puertas y ventanas enmarcadas, lo que da su encanto a esta arquitectura". El proyecto ha salido de estas referencias y de hecho, las traducen con un lenguaje arquitectónico renovado, donde algunos ven la expresión de un cierto "post-modernismo" regionalista.
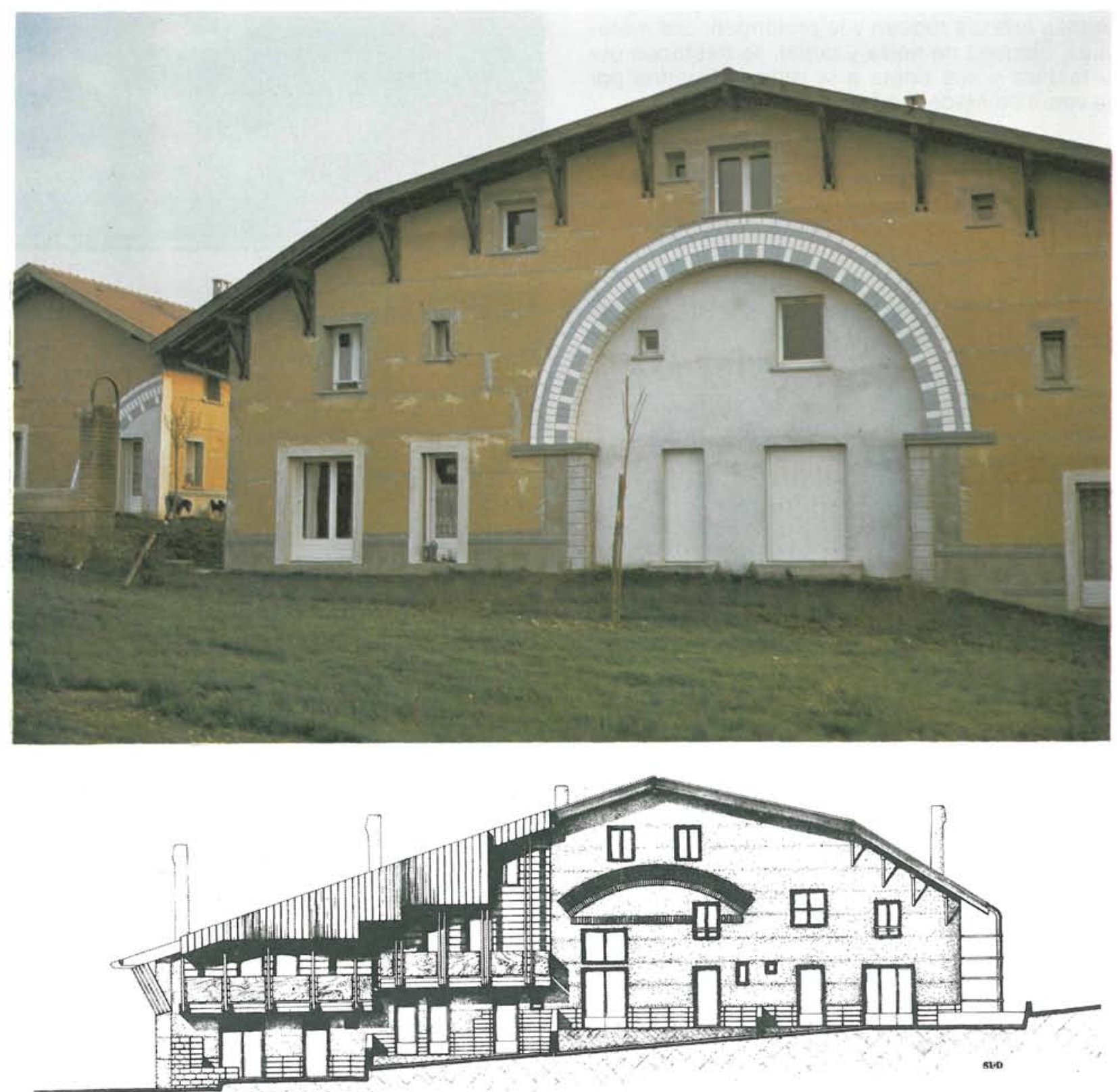


\section{8}

\section{CINCO VIVIENDAS DE BLOQUES DE TIERRA VIBRO-COMPACTADA Y ESTABILIZADA, DOMINADOS POR UNA TORRE-PALOMAR DE CINCO PISOS DE TAPIAL NO ESTABILIZADO}

Arquitecto: Jean Vincent Berlottier

Destinado a albergar especialmente una zona del Ecomuseo de Nord-Dauphiné.

Este proyecto ha sido objeto de un concurso complementario ganado por este arquitecto de Bourg-en-Bresse (Ain). El proyecto acusa las incitaciones de emplazamiento en la cima de la colina dominante del "Domaine de la Terre". Propone una masa compacta para las viviendas y esbelta para la torre de tapial. Se asemeja en su aspecto al concepto del poblado preexistente y que se encarama en el "campanario". Los cinco pisos de las torres se anclan al suelo por las viviendas que los rodean y la prolongan. Los materiales, bloques de tierra y tapial, se destacan por su textura y sus tintes a la vista, realzados por las vetas de madera en colores pastel.

La torre de tapial será un anexo del "Ecomuseo de Nord-Dauphiné"; ha sido financiado por la «Fondation des Pays de France».

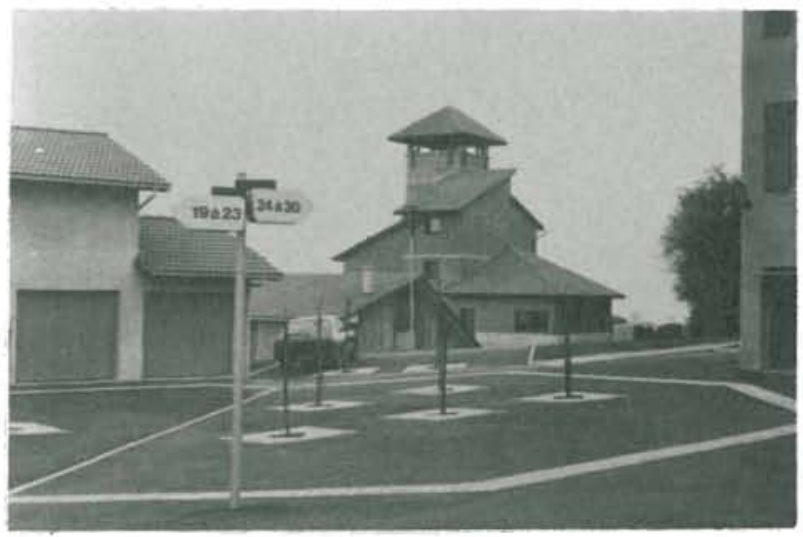

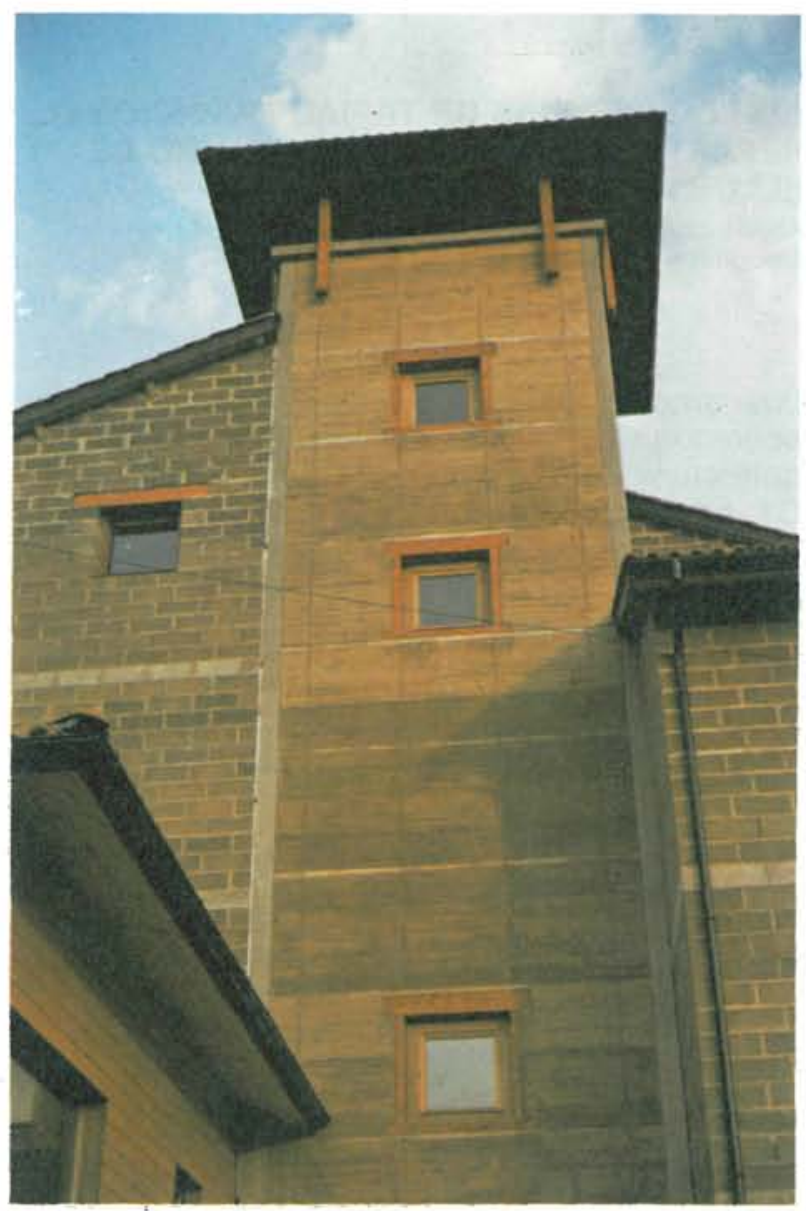

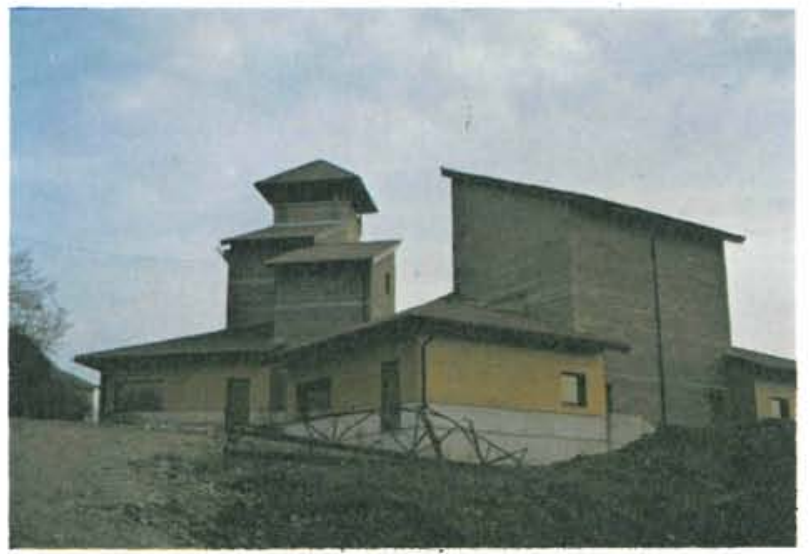

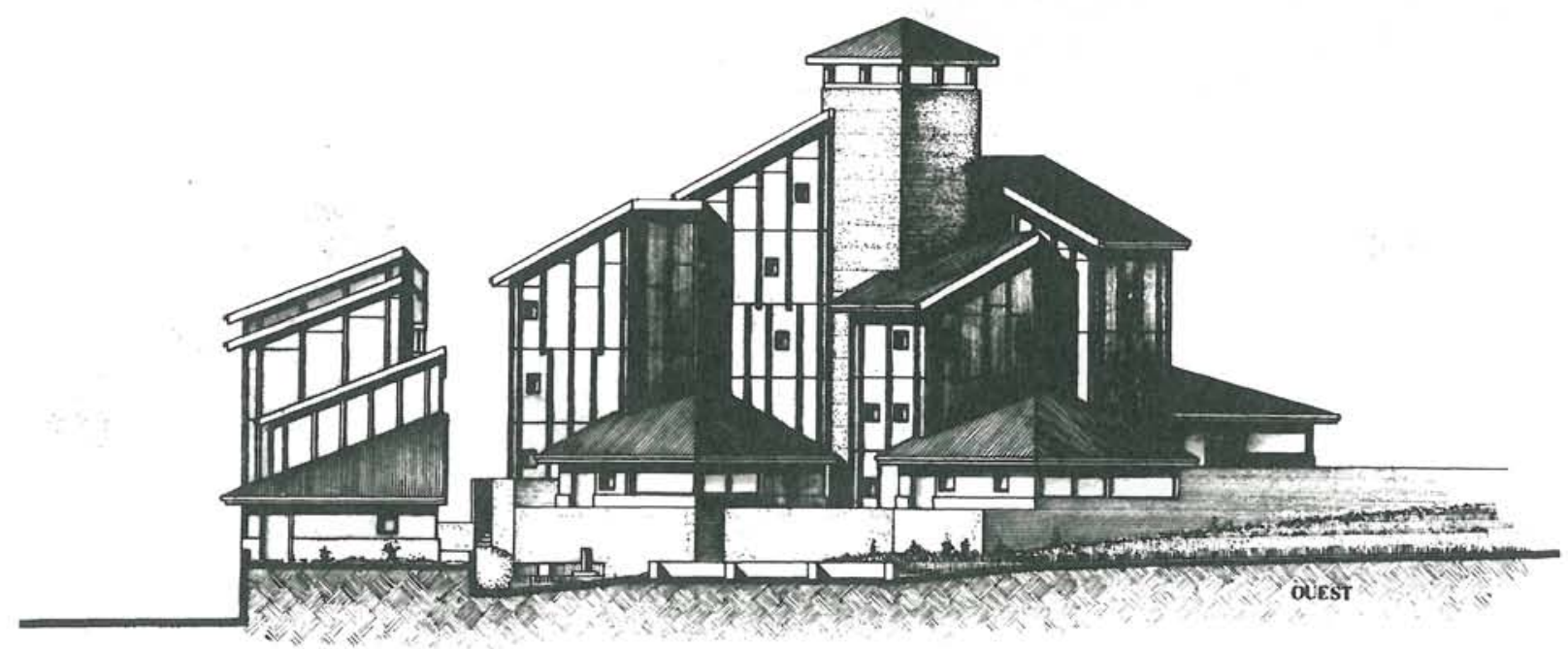

